\title{
Enfermagem no cuidado paliativo a criança com leucemia
}

\author{
Nursing in palliative care for children with leukemia
}

\section{Enfermería em cuidados paliativos para niños com leucemia}

\begin{abstract}
Valéria Lélis da Silva Ferreira ${ }^{1 *}$, Daniela Brito de Siqueira ${ }^{1}$, Joice Pinheiro dos Santos ${ }^{1}$, Katiúscia Oliveira de Paulo ${ }^{1}$, Larissa dos Santos e Santos ${ }^{1}$, Letícia de Souza Alves ${ }^{1}$, Mirela Kely de Souza Ferreira $^{1}$, Rodolfo Fernandes Oliveira da Costa ${ }^{1}$, Thiago Augusto Batista Trindade ${ }^{1}$, Graciana de Sousa Lopes ${ }^{1}$.
\end{abstract}

\section{RESUMO}

Objetivo: Descrever através de uma revisão narrativa a atuação da enfermagem no cuidado paliativo a criança com leucemia, identificar ações voltadas para a melhora da qualidade de vida e da criança com leucemia, relatar a pratica no cuidado paliativo a criança portadora de leucemia. Revisão Bibliográfica: os cuidados paliativos são ferramentas utilizadas na abordagem voltada para a melhoria da qualidade de vida de paciente e de seus familiares acerca de questões que envolvem doença terminal, preconizando a prevenção e o alívio do sofrimento, reconhecendo, analisando e tratando tanto a dor como outros problemas de cunho físico, psicossocial e espiritual. Essa metodologia propõe uma alteração no modelo de cuidado aos pacientes terminais, mudando paradigmas que curabilidade para o cuidado em si, os quais não tem objetivo de curar, mas sim de trazer um conforto ao paciente e a família dele. Considerações Finais: Os cuidados paliativos têm o objetivo de minimizar o sofrimento do indivíduo frente a uma doença sem chances de cura, grarantido qualidade de vida, conforto e dignidade ao paciente, o enfermeiro que atua nos cuidados paliativos da criança com câncer precisa orientar tanto o paciente quanto a sua família sobre os cuidados a serem feitos.

Palavras-chave: Leucemia, Cuidados paliativos, Pediatria, Enfermagem.

\begin{abstract}
Objective: To describe, through a narrative review, the role of nursing in palliative care for children with leukemia, to identify actions aimed at improving the quality of life and for children with leukemia, to report the practice of palliative care for children with leukemia. Bibliographic Review: palliative care is a tool used in the approach aimed at improving the quality of life of patients and their families about issues involving terminal illness, advocating the prevention and relief of suffering, recognizing, analyzing and treating both pain as well as other physical, psychosocial and spiritual problems. This methodology proposes a change in the model of care for terminally ill patients, changing paradigms that curability for the care itself, which has no objective of curing, but of bringing comfort to the patient and his family. Final Considerations: Palliative care aims to minimize the individual's suffering in the face of a disease with no chance of cure, ensuring a quality of life, comfort and dignity to the patient. The nurse who works in the palliative care of children with cancer needs to guide patient and family about the care to be taken.
\end{abstract}

Keywords: Leukemia, Palliative care, Pediatrics, Nursing.

1 Centro Universitário Fametro (CEUNI-FAMETRO), Manaus - AM.
^E-mail: valeria16121998@gmail.com

SUBMETIDO EM: 10/2020 


\section{RESUMEN}

Objetivo: Describir, a través de una revisión narrativa, el papel de la enfermería en los cuidados paliativos del niño con leucemia, identificar acciones dirigidas a mejorar la calidad de vida y del niño con leucemia, informar la práctica de cuidados paliativos en el niño con leucemia. Revisión Bibliográfica: los cuidados paliativos son una herramienta utilizada en el abordaje orientado a mejorar la calidad de vida de los pacientes y sus familias sobre temas relacionados con enfermedades terminales, abogando por la prevención y alivio del sufrimiento, reconociendo, analizando y tratando tanto el dolor. así como otros problemas físicos, psicosociales y espirituales. Esta metodología propone un cambio en el modelo de atención al enfermo terminal, cambiando paradigmas que la curabilidad para el cuidado en sí, que no tiene como objetivo curar, sino brindar consuelo al paciente y su familia. Consideraciones Finales: Los cuidados paliativos tienen como objetivo minimizar el sufrimiento del individuo ante una enfermedad sin posibilidad de cura, asegurando una calidad de vida, comodidad y dignidad al paciente, la enfermera que trabaja en los cuidados paliativos del niño con cáncer debe orientar tanto a la paciente y familia sobre los cuidados que se deben tener.

Palabras clave: Leucemia, Cuidados paliativos, Pediatría, Enfermería.

\section{INTRODUÇÃO}

Nomeia-se câncer o conjunto de centenas de doenças que apresentam como característica peculiar o crescendos celular desordenado que invadem tecidos e órgão. Tais células, ao sofrerem rápida divisão, pendem-se a ser demasiadamente agressivas e incontroláveis, ocasionando a formação de tumores, que podem verter para outras regiões do corpo, possuindo alta taxa de morbidade mundial, tendo uma estimativa, no campo da oncologia pediátrica, de aproximadamente 12,6 mil novos casos, sendo esse diagnóstico a primeira causa de óbito de adolescentes e de crianças com mais de 01 ano de vida (BRASIL, 2019; PACHECO CL e GOLDIM JR, 2019).

Segundo Nascimento CAD, et al. (2016) no que se refere as leucemias, define-se esta como sendo uma neoplasia maligna resultante de uma falha na hematopoese, causando um comprometimento quanto ao desenvolvimento e funcionamento nas células sanguíneas devido a proliferação e acúmulo de células percussoras no caminho. O câncer infantil é considerado uma doença rara, e que o tratamento é longo e traumático tanto para a criança como para os envolvidos no processo, sejam eles familiares ou profissionais de saúde. Nem para todos os casos existe uma possibilidade de cura, apesar dos avanços tecnológicos que visam a cura, o que leva a um padecimento emocional, psicossocial, espiritual e físico durante a terapêutica, porém o público infantil em benefícios no que cerne ao cuidado paliativo (MARTINS GB e HORA SS, 2017; SOUSA ADR, SILVA LF e PAIVA ED, 2019).

A OMS (2019) entende o termo cuidado paliativo (CP) como um recurso terapêutico que visa a melhoria na qualidade de vida dos pacientes, sejam eles adultos ou crianças, e das famílias que passam por problemas oriundos de patologias que põe em risco a vida, promovendo a prevenção e o alivio do penoso sofrimento por meio da avaliação e identificação precoce e tratamento da dor e demais problemáticas físicas, psicossociais ou espirituais. O profissional enfermeiro, ao exercer suas atribuições frente a criança com quadro oncológico, padece de esgotamentos físico e emocional, podendo-o levar a manifestações de problemas de saúde ou, ainda, levá-lo a trocar de campo de atuação. Sendo assim, infelizmente a enfermagem, dentro do campo profissional, enfrenta eventos de mortes, deparando-se sempre com a perda e a dor, precisando, com isso, vencer esses acontecimentos, profissionalmente, firmando o fim da vida como um processo natural (PROENCIO CC, et al., 2017).

Diante disso, o presente estudo tem por objetivo descrever através de uma revisão narrativa a atuação da enfermagem no cuidado paliativo a criança com leucemia, identificar ações voltadas para a melhora da qualidade de vida e da criança com leucemia, relatar a prática no cuidado paliativo a criança portadora de leucemia e elucidar a importância da enfermagem frente aos cuidados paliativos da criança com leucemia, levando em conta que essa população de pacientes é frágil e exige da equipe cuidados específicos e individuais. Cada criança responderá de uma forma frente a doença, portanto, cabe a enfermagem prestar um cuidado personalizado e voltado para criança e seus familiares, trazendo dignidade e conforto para esse momento. 


\section{REVISÃO BIBLIOGRÁFICA}

\section{O Câncer}

O câncer tem como peculiaridade o descontrolado crescimento celular que ocasiona e um montante células chamado de neoplasia ou tumor, e é considerado uma enorme problemática em saúde pública, pois causa um impacto imensurável na vida dos indivíduos que por essa doença são acometidos, assim como na vida de seus familiares, nas pessoas mais próximas e nos profissionais de saúde. Por ser uma doença que leva a perda da integridade de seu portador, também gera impacto nos órgãos de prestação de serviço em saúde do público usuário, bem como mutilações corpóreas e finitude do ciclo vital causando, assim uma perspectiva negativa por revelar um ambiente de luta no período de terapêutica e tentativa de remissão da doença (MOREIRA D, 2015).

Entende-se o câncer como uma forte ameaça a vida e às atividades que 0 indivíduo desempenha no dia a dia, e a respeito da sua expectativa sobre o futuro. $O$ impacto que a doença traz ao cidadão deixa-o inerte e estagna sua existência e seu relacionamento com o mundo (NASCIMENTO CAD, et al., 2016).

Dentre as neoplasias malignas pode-se citar as leucemias e linfomas como os responsáveis em provocar alterações metabólicas no paciente que, com isso, faz com que o tempo de internação na unidade de saúde hospitalar seja mais prolongado somando-se a as terapêuticas que combatem as neoplasias acabem trazendo impacto direto na qualidade de vida dos pacientes oncológicos (CRUZ TC, et al., 2018).

\section{O Câncer na Infância}

Vieira APHS, et al., (2016) classificam o câncer infantil como toda doença neoplásica que atinge indivíduos cuja faixa etária seja menor que quinze anos, o câncer nesse momento da vida gera um grande conflito para essas crianças, pois, a doença muitas vezes não é compreendida por elas e por seus familiares, se fazendo necessário apoio psicológico para esses indivíduos, a internação também se torna um fator de grande estresse para essas crianças, pois, a retiram da sua rotina e ambiente habitual.

O câncer na infância é consideravelmente raro, porém vem se tornado, nos últimos anos, uma das principais causas de óbito entre crianças com faixa etária abaixo dos 15 anos. Não se pode negar que o avanço tecnológico bem como a valorização do diagnóstico precoce sobreleva os índices e sobrevida das crianças acometidas por câncer, porém também existem, dentre elas, as que não terão êxito em curar a neoplasia, então, diante disso, é indicada a terapêutica paliativa até sua terminalidade (SILVA AF, et al., 2011). Dentre as inúmeras neoplasias que acometem a criança estão as leucemias, os tumores cerebrais, os linfomas, os tumores renais, os sarcomas e outras, representando, assim, de 1 a $3 \%$ de cânceres na população (FRANÇA JRFS, et al., 2017).

Por décadas e décadas o câncer pediátrico era tido como uma doença de mau prognóstico, porém, na atualidade, já existe uma considerável possibilidade de cura, cujo percentual de sobrevida pode chegar a 70\% dos casos (MONTEIRO TA, et al., 2014). O câncer que atinge a população infantil é bem diferenciado do câncer observado na população adulta nas questões de sua freqüência e tipo histológico. Na criança essa doença atinge as células hematopoiéticas e os tecidos de sustentação, nos adultos ela atinge as células epiteliais que recobrem os órgãos dos diversos sistemas do corpo (VIEIRA APMS, et al., 2016)

A evolução da neoplasia pediátrica é variável em questões de tipo, idade do paciente e início da terapêutica. Sendo assim ela pode apresentar desde remissão dos sintomas até a cura, que é uma peculiaridade dos pacientes que deixam de apresentar os sintomas ou outras alterações no quadro clínico após dez anos da última atividade terapêutica. A forma recidiva da doença ocorre após o período de remissão e controle, quando ocorre a volta dos sintomas, requerendo um novo ciclo de tratamento, bem como preparação para cuidados paliativos, os quais são aplicados quando não se tem mais possibilidade tratamento para cura, podendo o óbito acontecer em qualquer estágio da doença (GURGEL LA e LAGE AMV, 2013).

$\mathrm{Na}$ atualidade, os exames imaginológicos mais utilizados para fins de diagnóstico e acompanhamento da evolução do câncer pediátrico, além do uso de marcadores tumorais, são o raio-x convencional, a ultrassonografia, a tomografia computadorizada e a ressonância magnética (MULTTI CF, et al., 2010). 
A terapêutica é desenvolvida de forma individualizada e personalizada levando em consideração o metabolismo da criança, a biologia tumoral. A abordagem do tratamento é direcionada para cura até em doença disseminada, o que eleva a taxa de sobrevida do paciente e minimiza os efeitos oriundos do tratamento tardio. Para que a terapia seja eficaz é necessário que se observe o controle local e sistêmico da doença e o suporte para as reações adversas. O manejo do tratamento é feito à base de quimioterapia, cirurgia, radioterapia e suporte clínico (MONTEIRO TA, et al., 2014).

\section{Leucemias}

O INCA (2018) dispõe que a Leucemia é uma doença maligna, de origem desconhecida, que afeta os glóbulos brancos ocasionando, assim, o acúmulo de células defeituosas, ou seja, doentes, na medula óssea, subsistindo, com isso, as células normais. Classifica-se a Leucemia na pediatria em consonância com a linhagem celular atingida, onde a grande maioria dos casos envolve a mutação maligna de células chamadas progenitoras linfóides e em menor caso das células progenitoras mielóides, ou seja, aguda ou crônica (RODRIGUES RS, et al., 2015; SARAIVA DCA, et al., 2018).

Segundo o Instituto Nacional do Câncer (INCA) estimou que para cada ano do biênio 2018/2019, a LLA seja considerada a neoplasia mais frequente entre as crianças de até 5 anos, após essa idade, o risco declina lentamente até a faixa dos 20 anos, começando a aumentar lentamente após os 50 anos, estima-se que no Brasil cerca de 10.800 novos casos, sendo 5.940 em homens e 4.860 em mulheres no ano de 2018.

Segundo Nascimento CAD, et al. (2016) corroboram que as células sanguíneas são produzidas na medula óssea ou, ainda, nos gânglios linfáticos e baço e que, dependendo da linhagem celular tem-se a leucemia mielóide e a leucemia linfóide, sendo que as suas evoluções podem ser agudas ou crônicas, resultando, com isso, em quatro tipos possíveis: Leucemia Mieloide Aguda (LMA) ou crônica (LMC) e Leucemia Linfoide Aguda (LLA) ou crônica (LLC).

De acordo com INCA (2018) o tratamento para a poliquimioterapia tem indicação de ser realizado por etapas, sendo a primeira com finalidade de obtenção e remissão completa, ou seja, de uma forma que aparente normalidade duradoura. Conforme o mesmo autor as pesquisas comprovam que restam no organismo costuma ficar circulante muitas células leucêmicas, sendo necessário a continuação do tratamento para que não ocorra recaídas ou recidivas, e nas outras etapas o tratamento varia de acordo com o tipo de célula afetada.

\section{Cuidados Paliativos}

Para Brito MA, et al. (2015) os cuidados paliativos são ferramentas utilizadas na abordagem voltada para a melhoria da qualidade de vida de paciente e de seus familiares acerca de questões que envolvem doença terminal, preconizando a prevenção e o alívio do sofrimento, reconhecendo, analisando e tratando tanto a dor como outros problemas de cunho físico, psicossocial e espiritual. Essa metodologia propõe uma alteração no modelo de cuidado aos pacientes terminais, mudando paradigmas que curabilidade para o cuidado em si, os quais não tem objetivo de curar, mas sim de trazer um conforto ao paciente e a família dele.

$O$ cuidado paliativo tem ênfase na melhora do bem-estar dessas crianças, fornecendo apoio aos seus familiares e atentando para sintomas psicológicos e fornecendo apoio no momento do falecimento e durante a perda do seu ente. Essa modalidade de cuidado centra-se nos elementos sociais, psicológicos, emocionais e místicos, para essas crianças e adolescentes com doenças terminais (MOREIRA D, 2018; MENDES EC e VASCONCELLOS LCF, 2015).

Sendo assim os cinco princípios da assistência paliativa que são: 1. Veracidade, 2. Prevenção, 3. Não abandono, 4. Conduta ética e legal 5. Proporcionalidade. Vale ressaltar que a assistência paliativa também adentra o ambiente domiciliar, assim como se deve vislumbrar que é de suma importância que seja prestada ao paciente um atendimento humanizado, respeitando sua autonomia e os princípios do cuidar paliativamente (SILVA CF, et al., 2019).

A enfermagem, no contexto da oncologia e CP, integra a equipe interdisciplinar de cuidados no processo de morte e morrer, com o objetivo de ajudar o paciente e a sua família a viverem o mais ativamente possível esses últimos momentos, afirmando a vida e percebendo a morte como parte do ciclo vital (CUNHA AR, et al., 2016). 
Tais cuidados requerem algumas habilidades do profissional como vocação para o cuidado; certo altruísmo; empatia e compreensão; capacidade de escuta; sinceridade/honestidade; habilidade na comunicação: equilíbrio e maturidade pessoal, especialmente diante da morte; respeito aos valores, crenças e cultura do paciente/família e flexibilidade (CUNHA AR, et al., 2016).

Os CP devem ser iniciados de imediato após o diagnóstico da doença, para assim permitir o acompanhamento perante as medidas terapêuticas com foco na cura do paciente, porém se esta medida não for atingida, inicia-se as medidas paliativas exclusivas, e o cuidado principal é mediando perante a cura (SILVA AF, et al., 2015).

A assistência em CP requisita atuação qualificada, na qual a partilha dos conhecimentos técnicos e científicos vai culminando na apreensão das dimensões éticas da vida, do trabalho, do homem, da saúde e das relações. Esse modelo de assistência exige dos profissionais novos competências que remetam direta, mas não exclusivamente, à pesquisa, à produção de conhecimento e às alternativas de intervenção na realidade social (MARTINS GB e HORA SS, 2017).

Alcântara EH, et al. (2018) e Alves RSF, et al. (2019) caracterizam os CP como cuidar ativa e totalmente os pacientes nas quais a doença não apresenta resposta positiva a terapêutica de cura. Com isso, os CP têm o objetivo de ofertar a máxima qualidade de vida possível para os pacientes e familiares, por intermédio do controle da dor e de outros sintomas, bem como de problemas psicossociais e espirituais direcionados a quadros clínicos avançados da doença, progressivo e incurável. Os CP também são empregados quando não existe tratamento específico, na existência de inúmeros sintomas de elevada intensidade, múltiplos, multifatoriais e mutantes. Ainda, em grandes impactos emocionais que atinjam o doente, a família e os cuidadores, com relação específica a eminência ou não de morte breve, e prognóstico de vida com período inferior a seis meses.

Sob a ótica que os princípios dos CP são a oferta de qualidade de vida e controle da dor e sofrimento, compreende-se que a equipe multidisciplinar precisa estar capacitada para poder atender o paciente e a família em sua totalidade. A minimização das angústias, o controle da dor e o alívio dos sintomas contribuem, portanto, para uma melhor qualidade de vida, independentemente do tempo restante (PICOLLO DP e FACHINI M, 2018).

Uma questão de suma relevância e primordialidade para o estabelecimento de uma relação entre o paciente, sua família e a equipe de enfermagem está a prestação da assistência ao paciente em sua totalidade, preservando sua individualidade, fazendo uso de mecanismos que levem a uma cumplicidade partindo de uma comunicação segura e eficaz, de forma humanizada (ANDRADE CG, et al., 2017.) A enfermagem pode promover o conforto à criança frente a situação de estar doente, proporcionando alívio a dor e sensação de bem-estar através da palavra, contato físico e apoio psicológico para uma morte digna (SOUSA ADRS, et al., 2019).

Soares VA et al. (2014); entendem que a atividade de brincar é uma forma de promover uma assistência humanizada à criança com neoplasia em $\mathrm{CP}$, sendo de suma importância integrar essa modalidade aos cuidados a essas crianças. Já Multti CF et al. (2010); enfatizam que a atividade de brincar propicia um período de hospitalização mais suportável e menos traumático para a criança doente, pois a forma lúdica produz relaxamento, alivia a tensão e expressa sentimentos, fazendo com que o estresse da separação diminua, bem como o pesar em estar longe de casa, levando a criança a se sentir mais segura apesar de estar em um ambiente estranho.

De igual modo, os autores França JRFS et al. (2017); relatam que atividades lúdicas como desenvolver brincadeiras são estratégias importantíssimas para o enfrentamento de doenças, pois ofertam a criança a comunicação e a expressão ativa de seus sentimentos, pois é brincando que a criança transforma seu ambiente redefinindo a realidade na qual está vivendo, buscando, com isso, amenizar o sofrer por ter que vivenciar uma doença que traz ameaça a vida.

Souza CTF et al. (2018); o emprego de outras formas terapêuticas para no CP para manter a qualidade de vida também podem ser empregadas como posicionamento, relaxamento, massagem, bem como e outras medidas além da eficácia em associar a da terapia farmacológica para o alívio da dor com atividades lúdicas, de arte, leitura, música e atividades recreativas, bem como acolhimento e escuta por parte do profissional. 
Uma devastadora carga sintomatológica aflige o paciente em estado terminal, são eles de cunho físico, emocional e psicológico, e necessitam que precocemente sejam adotadas ações terapêuticas ativas e dinâmicas, contudo, deve-se respeitar as limitações do paciente acerca da situação de incurabilidade (BRASIL, 2018).

Para Silva AF et al. (2015); a equipe envolvida no CP deve ser dedicada e cautelosa, visando uma assistência que tenha como base o cuidado humanizado, respeitando a individualidade de cada paciente, pois o CP oportuniza segurança, conforto e confiança aos pacientes. Apesar do doente estar inserido nesse tipo de cuidado, a sua família possui um papel fundamental em conjunto com a equipe para que possa receber um suporte psicológico e emocional. Também é necessário que os profissionais envolvidos sejam capacitados e promovam ações que levem a melhoria no CP direto ao paciente em terminalidade de vida.

Diante da incurabilidade da doença, o enfermeiro deve manifestar o respeito pela vida do doente comprometendo-se em proporcionar proteção frente a vulnerabilidade, iniciando o CP na oportunidade em que 0 cuidado curativo deixa de ser o propósito principal. Atividades terapêuticas que não visam curar amenizam o sofrimento e levam conforto ao paciente, porém, ao se falar em estado terminal sem possibilidade de cura, o foco assistencial é deixado de lado.

Sendo assim, o processo do cuidar ao paciente, merece respeito aos valores e crenças destes indivíduos, da forma que ocorra preservação da dignidade humana, a ética e empatia, na busca do saber e do fazer de forma correta (SILVEIRA NR, et al., 2016).

\section{CONSIDERAÇÕES FINAIS}

Os cuidados paliativos tem o objetivo de minimizar o sofrimento do indivíduo frente a uma doença sem chances de cura, garantindo qualidade de vida, conforto e dignidade ao paciente, o enfermeiro que atua nos cuidados paliativos da criança com câncer precisa saber orientar tanto o paciente quanto a sua família sobre os cuidados a serem feitos, portanto, é preciso que o enfermeiro envolvido saiba educar em saúde, de maneira clara e objetiva, e ser prático e claro em suas ações, visando sempre o bem estar dos seus clientes, elevando, assim, a qualidade de vida e saúde dos seus pacientes, independente do diagnóstico dado a eles. O controle da dor dos pacientes terminais é o paliativo, e a dor faz parte do processo fisiopatológico terminal da doença, dessa forma o enfermeiro também é inserido na busca de propiciar o maior conforto a criança em seus momentos finais.

\section{REFERÊNCIAS}

1. AlCANTARA EH, et al. Percepção dos profissionais da equipe de enfermagem sobre o cuidar de pacientes em cuidados paliativos. Revista de Enfermagem do Centro-Oeste Mineiro, 2018; 8(2673): 1-7.

2. ALVES RSF, et al. Cuidados Paliativos: Alternativa para o Cuidado Essencial no Fim da Vida. Psicol. cienc. Prof, Brasília, 2019; 39(185734): 1-15.

3. ANDRADE CG, et al. Cuidados paliativos e comunicação: estudo com profissionais de saúde do serviço de atenção domiciliar, Rev. Fund. Care Online, 2017; 9(1): 215-221.

4. AVANCI BS, et al. Cuidados paliativos a criança oncológica na situação do viver/morrer: a ótica do cuidar em enfermagem. Esc. Anna Nery, 2009; 13(4): 708-716.

5. BETANCUR MAL., Cuidados al final de la vida: una oportunidad para fortalecer el patrón emancipatorio de enfermería. Av. Enferm. Bogotá, 2015; 33(1): 124-132.

6. BRASIL, Ministério da saúde. O que é câncer. 2019, Rio de Janeiro: INCA.

7. BRASIL, Ministério da Saúde. Cuidados Paliativos no SUS. A Portaria no $3.519 / \mathrm{GM} / \mathrm{MS}$, de 29 de outubro de 2018 , Diário oficial da união, 2018. 1677-7042.

8. BRITO MA, et al. Cuidados paliativos em pediatria: um estudo reflexivo. Rev. Enferm. 2015; 9(3): 7155-7160.

9. CARMO SA.; OLIVEIRA ICS. Criança com Câncer em Processo de Morrer e sua Família: Enfrentamento da Equipe de Enfermagem. Revista Brasileira de Cancerologia, 2015; 61(2): 131-138.

10. CRUZ TC, et al. Avaliação da qualidade de vida de pacientes com leucemia e linfoma hospitalizados. Rev. Pesqui. Fisioter, 2018; 8(1): 94-100.

11. CUNHA AR, et al. Pacientes oncológicos e em cuidados paliativos: o perfil dos profissionais enfermeiros e suas relações. Anais do IX Fórum Mineiro de Enfermagem. 2016; 1(9): 14-22.

12. FRANÇA JRFS, et al.; Vivência de crianças com câncer sob assistência paliativa em uma casa de apoio. REME, 2017; 21: 1-8. 
13. GOMES MIGI, Cuidados paliativos: relação eficaz entre equipe de enfermagem, pacientes oncológicos e seus familiares. Rev. Rede cuid. Saúde 2019; 13(2): 60-70.

14. GUIMARÃES TM, et al. Cuidados paliativos em oncologia pediátrica na percepção dos acadêmicos de enfermagem. Esc Anna Nery, 2016; 20(2): 261-267.

15. GURGEL LA; LAGE AM. V. Cuidados paliativos em oncologia pediátrica: uma perspectiva de atuação psicológica. Rev. SBPH, 2013. 16(1): 141-149.

16. HERMES HR; LAMARCA ICA. Cuidados paliativos: uma abordagem a partir das categorias profissionais de saúde. Ciência \& Saúde Coletiva, 2013; 18(9): 2577-2588.

17. MABUCHI AS, et al. O significado dos cuidados paliativos para os pais de uma criança com câncer. Saúde Coletiva, 2010; 7(45): 270-276.

18. MARTINS GB; HORA SS. Desafios à Integralidade da Assistência em Cuidados Paliativos na Pediatria Oncológica do Instituto Nacional de Câncer José Alencar Gomes da Silva. Revista Brasileira de Cancerologia 2017; 63(1): 29-37.

19. MENDES EC; VASCONCELLOS LCF. Palliative cares on cancer and the doctrinal principles of SUS. Saúde em Debate, 2015; 39(106): 881-892.

20. MONTEIRO TA, et al. A atuação do enfermeiro junto à criança com câncer: cuidados paliativos. Rev enferm UERJ, 2014; 22(6): 778-783.

21. MOREIRA D, et al. Paciente oncológico em estado terminal: cuidados paliativos como medida de conforto. Rev. equilibrio corporal e saúde, 2015; 9(10): 32-38.

22. MULTTI CF, et al. Assistência à Saúde da Criança com Câncer na Produção Científica Brasileira. Revista Brasileira de Cancerologia, 2010; 56(1): 71-83.

23. NASCIMENTO CAD, et al. Leucemia mieloide aguda (Ima): as condições psicológicas do paciente adulto. Psicol. rev. Belo Horizonte, 2016; 22(2): 336-355.

24. PACHECO CLR. Percepções da equipe interdisciplinar sobre cuidados paliativos em oncologia pediátrica. Rev. bioét.; 2019; 27(1): 67-75.

25. PERONDI BLB, et al., A enfermagem no cuidado paliativo de crianças com leucemia linfoide aguda terminal. Revista Ciências da Saúde e Educação IESGO. 2019; 1(1): 29-36.

26. PICOLLO DP; FACHINI M. A atenção do enfermeiro ao paciente em cuidado paliativo. Rev. Ciênc. Méd. 2018; 27(2): 85-92.

27. PROENCIO CC, et al. Síndrome de Burnout em trabalhadores da Enfermagem que são estudantes da graduação. Revista Saúde e Desenvolvimento, 2017; 11(6): 102-120.

28. RODRIGUES RS, et al. ACUTE MYELOID LEUKAEMIA: LABORATORY FEATURES. Visão Acadêmica, 2015; 16(2): 1-14.

29. SARAIVA DCA, et al.Tendência de mortalidade por leucemias em crianças e adolescentes nas capitais dos estados brasileiros: 1980-2015. Epidemiol. Serv. Saúde, 2018, Brasília; 27(3): 1-13.

30. SEKI NH, GALHEIGO SM. O uso da música nos cuidados paliativos: humanizando o cuidado e facilitando o adeus. Interface Comunicação, Saúde e Educação, 2010; 14(33): 273-284.

31. SHOJI S, et al. Proposals for improving working conditions at anout patient clinic: the nursing stand point. Esc Anna Nery [Internet]. 2016; 20(2): 303-309.

32. SILVA AF, et al. Cuidados paliativos em oncologia pediátrica: percepções, saberes e práticas na perspectiva da equipe multiprofissional. Rev. Gaúcha Enferm. Porto Alegre, 2015; 36(2): 56-62.

33. SILVA AF, et al. A família da criança oncológica em cuidados paliativos: o olhar da equipe de enfermagem. Cienc. Cuid Saúde, 2011; 10(4): 820-827.

34. SILVA CF, et al. Cuidadores formais e assistência paliativa sob a ótica da bioética. Rev. Bioét, 2019, Brasília; 27(3): 535-541.

35. SILVA LS, et al. Quality of Life of Patients with Advanced Cancer in Palliative Therapy and in Palliative Care. Aquichan, Bogotá, 2019; 19(3): 1-14.

36. SILVEIRA NR, et al. Cuidado paliativo e enfermeiros de terapia intensiva: sentimentos que ficam. Revista Brasileira de Enfermagem, 2016; 69(6): 1074-1081.

37. SOARES VA, et al. The use of playing by the nursing staff on palliative care for children with câncer. Rev Gaúcha Enferm, 2014; 35(3): 111-116.

38. SOUSA ADRS, et al. Nursing interventions in palliative care in Pediatric Oncology: an integrative review. Rev. Bras. Enferm, 2019; 72(2): 531-540.

39. SOUZA CTF, et al. Cuidados paliativos pediátricos: análise de estudos de enfermagem. Rev. Enferm, 2018; 12(5): 1409-1422.

40. VIEIRA APMS, et al. Assistência de enfermagem na oncologia pediátrica. Rev. Eletrôn. Atualiza Saúde, 2016; 3(3): 67-75. 